\title{
EVALUATION OF PHARYNGEAL AIRWAY VOLUME USING CONE - BEAM COMPUTED TOMOGRAPHY - CASE REPORT
}

\author{
Ştefania Dinu1, Emilia Brândușa Brăilă², Răzvan Mihai Horhat3, Mariana Păcurar4, Hajosman \\ Isam Osma ${ }^{4}$, Vlad Grigore ${ }^{4}$, Simina Boia ${ }^{5}$, Eugen-Radu Boia ${ }^{6}$, Magda Mihaela Luca ${ }^{1}$, Mălina \\ Popa $^{1}$
}

\section{Abstract}

Background: Visualization and calculation of the airway dimensions are important because an increase of airway resistance may affect the development of dentofacial structures by altering the breathing pattern of growing patients. The aim of this study is to shed light on the resources provided by cone - beam computed tomography in the diagnosis of possible physical barriers that may reduce upper airway permeability by presenting one case report. Material and method: A patient clinically diagnosed with oral respiratory syndrome initially underwent a profile teleradiography and orthopantomography. For a better assessment of the airway space, it was recommended to perform a cone - beam computed tomography. Results: On demand 3D software program automatically provides the total area and volume of any predefined region as well as the location and size of the narrowest portion of airspace. Conclusions: On demand 3D software allows rapid segmentation of the upper respiratory tract. Segmentation can be checked on 2D sections (axial, frontal and sagittal). Three - dimensional measurements of airway volume and most constricted surface can be accurately performed.

Keywords: Cone - Beam Computed Tomography, teleradiography, pediatric dentistry, upper airway.

\section{Introduction}

Cone beam computed tomography (CBCT) is probably one of the most revolutionary innovations in the field of pediatric dentistry and offers a new platform for orthodontic diagnosis and treatment planning.

Nowadays, the use of cone beam computed tomography $(\mathrm{CBCT})$ is increasing in orthodontic practice due to its $3 \mathrm{D}$ diagnostic ability with the continued reduction in cost and radiation exposure. [1]

Different three - dimensional (3D) imaging modalities are available to investigate airway morphology and the surrounding soft tissues. This allows for the quantification of volumetric, area and linear measurements. [2]

Upper airway space assessment is a routine procedure in orthodontic diagnosis and treatment planning. Most studies have been based on profile teleradiography because such radiographs are part of the usual records for good planning of orthodontic treatment. Although it provides a wealth of information, teleradiography is limited in the sense that it produces two - dimensional images (height and depth) of a three - dimensional structure, thus preventing the correct assessment of the size and complexity of this structure.

Although numerous methods with 2-dimensional (2D) cephalograms, providing limited data such as linear and angular has been proposed for upper airway studies, there were studies that evaluate the airway have introduced the use of CBCT, which made the 3D diagnosis of the patient became more accessible in dentistry. The segmentation of the airway can be done manually or automatically. Manual segmentation seems to be the most accurate method and allows for the most operator control. [2]

${ }^{1}$ Department of Pediatric Dentistry, Faculty of Dental Medicine, "Victor Babeș" University of Medicine and

Pharmacy Timisoara, Romania, Pediatric Dentistry Research Center (Pedo-Research)

${ }^{2}$ Department of General Dentistry of Municipal Emergency Clinical Hospital, Timișoara, Romania

${ }^{3}$ Department of Endodontics, Faculty of Dental Medicine, "Victor Babes" University of Medicine and

Pharmacy Timisoara, Romania, TADERP Research Center

${ }^{4}$ Department of Orthodontics, Faculty of Dental Medicine, University of Medicine and Pharmacy Targu Mures, Romania

${ }^{5}$ Department of Periodontology, Faculty of Dental Medicine, "Victor Babeș" University of Medicine and Pharmacy, Timișoara

${ }^{6}$ Department of ENT, Faculty of Medicine, "Victor Babeș" University of Medicine and Pharmacy, Timișoara

Email: dinu.stefania@umft.ro; braila.emilia@umft.ro; razvanhorhat@yahoo.com; mariana.pacurar@umftgm.ro, hajosman.isam@gmail.com; dr.ec.vlad.grigore@gmail.com; simina.boia@umft.ro; eugen.boia@umft.ro; popa.malina@umft.ro 
Manual segmentation needs the operator to delineate the airway slice by slice and render the data into a $3 \mathrm{D}$ volume for analysis. [3]

In the study about developing pediatric three-dimensional upper airway normative values, the authors, insisted that clinicians should utilize the proposedthere upper airway normative values for screening and assist in the timely diagnosis and management of pediatric sleep apnea. [4] CBCT made it possible to obtain 3D images with the volumes of all structures in the maxillofacial complex. With the use of specific software and procurement protocols based on individual needs, these digital volumetric scans can be transformed into multiple flat images (transverse, frontal and sagittal). The software also allows measurements of bone structures to be obtained, as well as 3D evaluation of soft tissues and shapes, volumes and features of the face and upper respiratory tract.

CBCT has contributed to bringing new information in orthodontics regarding the space of the upper respiratory tract, by acquiring three-dimensional images, which allows professionals to accurately determine the narrowest area, where the greatest resistance to air passage occurs.

\section{Objectives}

The aim of this study is to shed light on the resources provided by CBCT in the diagnosis of possible physical barriers that may reduce upper airway permeability by presenting one case report

\section{Materials and Methods}

A patient clinically diagnosed with oral respiratory syndrome initially underwent a profile teleradiography (figure 1) and orthopanthomography (figure 2). For a better assessment of the airway space, it was recommended to perform a CBCT examination covering the neck region. CBCT examination for airway assessment has a specific image acquisition protocol. Patients should be placed in the maximum intercuspid position, with the mid-sagittal plane perpendicular to the horizontal plane, the Frankfurt plane parallel to the horizontal plane. Upon completion of the CBCT examination, some manipulations may be performed using the software provided by the scanner manufacturer. The raw image (raw data) has been reconstructed to allow visualization of $3 \mathrm{D}$ reconstruction and multiplanar sections. These two-dimensional images can be viewed from any direction. The most commonly used are sagittal, coronal and transverse images. Images are best viewed with the help of specific tools. Images can be rotated and magnified to allow a better evaluation of a particular region. Images can also be played back from any angle, at any scale or position. We applied different filters thus allowing the differentiation between tissues with different densities and the use of transparency that allows the visualization of hard tissue among soft tissue. A linear measuring instrument is available, which can measure the height, depth and width of any part of the pharynx.

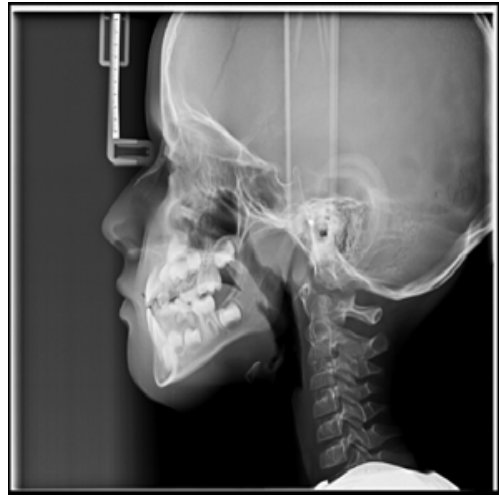

Fig. 1. Profile teleradiography of patient

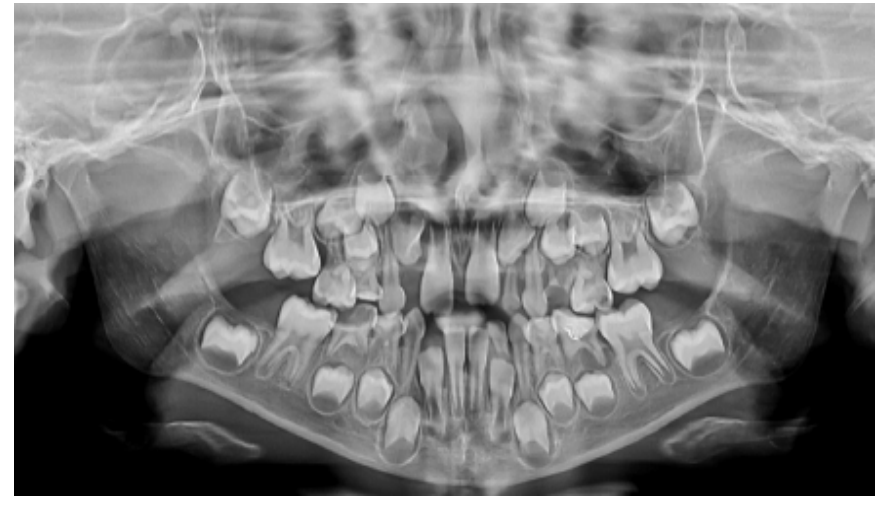

Fig. 2. Orthopantomography of patient
These images were transformed into DICOM (Digital Imaging and Communications in Medicine) files that allowed export to other assessment software, which in turn allow a wider range of resources useful in assessing airspace.

The On demand 3D version is an airway space analysis tool that not only allows you to evaluate the shape and contour of the upper airways in $3 \mathrm{D}$, but can also calculate the volume, sagittal areas and the smallest predefined crosssectional area in the airspace upper respiratory tract. It provides segmentation of upper airway space through rotatable and amplifiable images. The program has two threshold filters: for hard tissue and soft tissue, displaying the airway space along with the bone tissue or separately. To evaluate the images in the program, we first had to import the DICOM format files from the CBCT images. Once imported, the three-dimensional image of the patient's head was oriented in virtual space, similar to the cephalostat, so that the horizontal Frankfurt plane is parallel to the transverse plane, the medioagital plane coincides with the midline of the individual and the cranial plane is oriented so that it passes beyond from the lower edge of the orbits. In asymmetric cases, the orientation should be as close as possible to these reference plans. This virtual orientation allowed the head to rotate properly so that the bilateral 


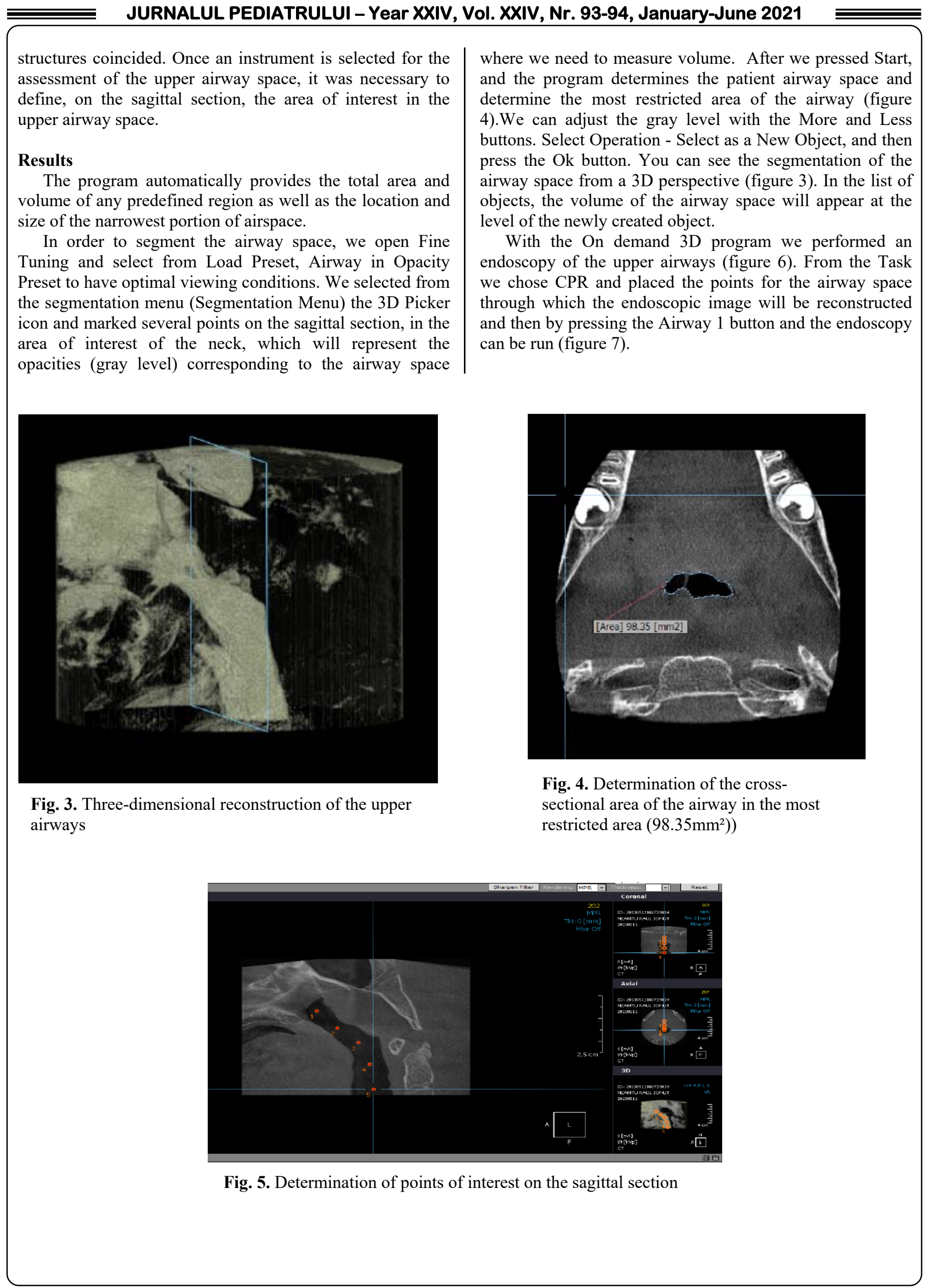




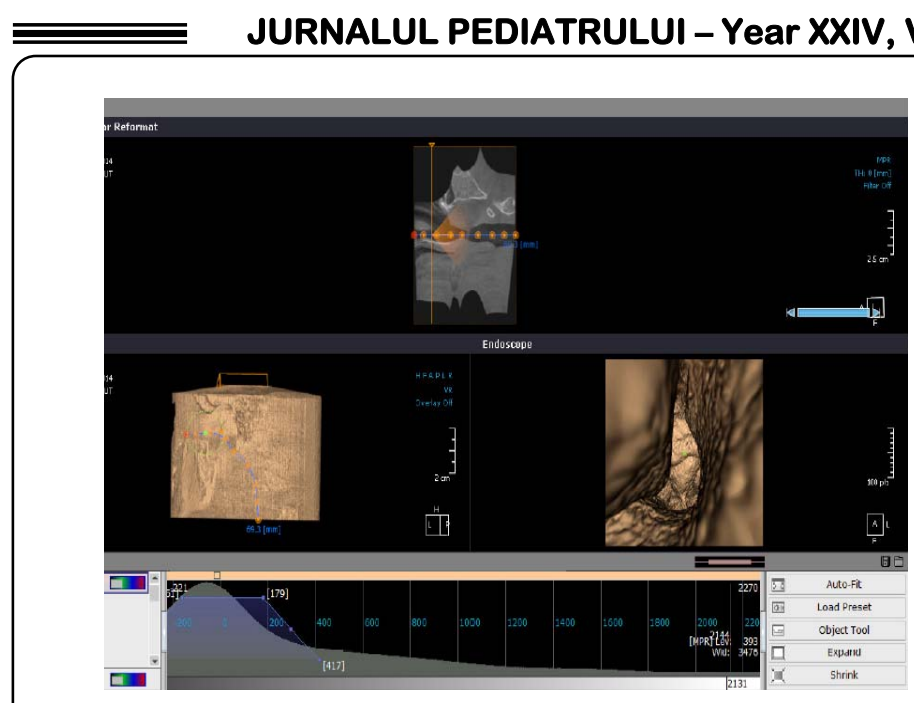

Fig. 6. The way to acquire an endoscope with the help of images provided by CBCT

\section{Discussion}

CBCT systems have been developed specifically for the maxillofacial region with the advantage of the reduced radiation doses compared with conventional CT. [5]

Clinicians who order or perform CBCT for orthodontic patients are responsible for interpreting the entire image volumes, just as they are responsible for interpreting all regions of other radiographic images that they order. [6]

With advances in medical care, the pharyngeal airway space of orthodontic patients is beginning to attract attention. The pharyngeal airway can be divided into three sections, namely the nasopharyngeal, oropharyngeal, and laryngopharyngeal airways. The nasopharyngeal and the oropharyngeal airways are demarcated by the retro palatal region of maxilla, whereas the oropharyngeal and the laryngopharyngeal airways are demarcated by the tip of the epiglottis. [7]

Over the past century, a large number of tests have been proposed to evaluate the upper respiratory tract on profile teleradiographs using linear and angular measurements and sagittal areas between cephalometric points. [8, 9] These points are defined by the overlapping projections of the different structures.

In the study about developing pediatric three-dimensional upper airway normative values, the authors, insisted that clinicians should utilize the proposedthere upper airway normative values for screening and assist in the timely diagnosis and management of pediatric sleep apnea. [4]

In a comparison between CT and profile teleradiography in the assessment of pharyngeal airspace, Aboudara et al. [10] found a significant correlation between the sagittal surface obtained by cephalometry and the volume obtained by CBCT, although the latter showed greater variability in patients with airway space similar to profile teleradiography. This was to be expected since only height and depth can be measured on profile teleradiography and therefore does not allow cross-examination (ie width).

Accuracy and reliability of airway measurements for volume and minimum area in CBCT images have been tested. Lenza et al. [11] had compared the linear, area, and

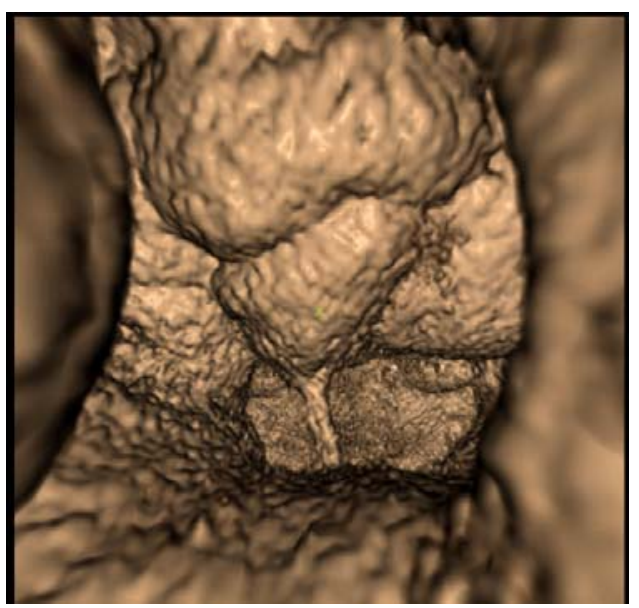

Fig.7. The endoscopy provided by CBCT

volumetric measurements by two examiners and found no significant differences.

Aboudara et al. [10] did that study to compare the nasopharyngeal airway size between a lateral head film and a CBCT scan in adolescent subjects and found that there is a significant positive relationship between nasopharyngeal airway size on a head film and its true volumetric size from a CBCT scan.

Ghoneima \& Kula [5] did had investigated the accuracy of CBCT airway measurements by scanning the actual volume of an airway model. The results of their study showed that the CBCT digital measurements of the airway volume and the minimum area of the airway are reliable and accurate. [3]

The size of the airspace and the morphology vary when the patient inhales and exhales. [12]

The acquisition time of CT scans is about $20-40$ seconds, too long for the individual to control their respiratory movements. Hopefully, in the near future, the time required will be shorter in order to prevent the patient's movements (breathing, swallowing and involuntary movements) from interfering with the results. Several imaging software programs are currently available for upper airway assessment. In addition to On demand 3D, Dolphin 3D, InVivo Dental, Mimics, OsirX, ITK-Snap, etc. can also be used.

Because the technology develops, the efforts to reduce exposure and to improve image quality have led to new innovations. These can include flat panel detectors with greater photon sensitivity, automatic exposure control with photon counting, customizable FOV collimation and image quality settings. Several studies have accumulated valuable data on technology assessment, craniofacial morphology in health and disease, treatment outcomes and efficacy of CBCT. Currently, the main limiting factor for widespread use of $\mathrm{CBCT}$ in orthodontics is the radiation dose especially in children. $\mathrm{CBCT}$ is a supplementary diagnostic aid with lot of radiation risk. It is not an essential diagnostic aid so it is unwise at present to make it mandatory for all patients. It is suggested that routine radiographs as well as $3 \mathrm{D}$ radiographs should not be prescribed routinely [14]. 
CBCT examinations must not be carried out unless a clinical examination have been performed. CBCT examinations must be justified for each patient.

\section{Conclusions}

CBCT allows clinicians to assess the airspace and surrounding structures and determine nasal, oro- and hypopharyngeal measurements, such as the most constricted area, volume, and smallest antero-posterior and lateral dimensions of the pharynx in patients with respiratory problems. It is also possible to assess changes that may be induced by the treatment modality itself, and to identify which patient could benefit from such treatment. CBCT will be able to guide orthodontic diagnosis and planning by showing clinicians about the effects of mechanotherapy applied to the airway space and the consequences of these effects. On demand 3D software allows rapid segmentation of the upper respiratory tract. Segmentation can be checked on 2D sections (axial, frontal, and sagittal). Three dimensional measurements of airway volume and most constricted surface can be accurately performed.

\section{Acknowledgments}

We thank Dr. Tudericiu Vlad for helping us with the On demand 3D software program and Dr. Ana Maria Hadarean for helping us with the aquisition of the data.

\section{References}

1. Garib DG, Calil LR, Leal CR, Janson G. Is there a consensus for CBCT use in Orthodontics? Dental Press J Orthod. 2014;19(5):136-49.

2. El H, Palomo JM. Measuring the airway in 3 dimensions: a reliability and accuracy study. Am J Orthod Dentofac Orthop. 2010;137(4 Suppl):S50 e1-9

3. Kamaruddin N, Daud F, Yusof A, Ezane Aziz M, Rajion ZA. Comparison of automatic airway analysis function of Invivo5 and Romexis software. PeerJ. 2019 Jan 23;7:e6319.

4. Masoud AI, Alwadei FH, Alwadei AH, et al. Developing pediatric three-dimensional upper airway normative values using fixed and interactive thresholds. Oral Radiol. 2020 Jan;36(1):89-99.

5. Ghoneima A, Kula K. 2013. Accuracy and reliability of cone-beam computed tomog- raphy for airway volume analysis. Eur J Orthod. 2013 Apr;35(2):256-61

6. Carter L, Farman AG, Geist J, et al. American Academy of Oral and Maxillofacial Radiology executive opinion statement on performing and interpreting diagnostic cone beam computed tomography. Oral Surg Oral Med Oral Pathol Oral Radiol Endod. 2008 Oct;106(4):561-2

7. Hiatt JL, Gartner LP. Textbook of head and heck anatomy. New York, NY: Appleton-Century-Crofts, 1982:48e56.

8. Alamri HM, Sadrameli M, Alshalhoob MA, Sadrameli M, Alshehri MA. Applications of CBCT in dental practice: a review of the literature. Gen Dent. 2012; 60(5):390-400,
9. Weissheimer A, Macedo de Menezes L, Sameshima GT, Enciso R, Pham J, Grauer DImaging software accuracy for 3-dimensional analysis of the upper airway. Am J Orthod Dentofacial Orthop. 2012 Dec;142(6):801-13.

10. Aboudara C, Nielsen I, Huang JC, Maki K, Miller AJ, Hatcher D. Comparison of airway space with conventional lateral headfilms and 3-dimensional reconstruction from cone-beam computed tomography. Am J Orthod Dentofacial Orthop. 2009 Apr;135(4):46879

11. Lenza MG, de O Lenza MM, Dalstra M, Melsen B, Cattaneo P. 2010. An analysis of different approaches to the assessment of upper airway morphology: a CBCT study. Orthod Craniofac Res. 2010 May;13(2):96-105.

12. Schwab RJ, Goldberg AN. Upper airway assessment: radiographic and other imaging techniques. Otolaryngol Clin North Am. 1998 Dec;31(6):931-68

13. Kapila S, Conley RS, Harrell Jr WE. The current status of cone beam computed tomography imaging in orthodontics. Dentomaxillofac Radiol. 2011 Jan;40(1):24-34.

14. Makhija PG. CBCT in orthodontics: current trends and caveats. Journal of Contemporary Orthodontics.2017 Mar; 1(2):01-11.

\section{Correspondence to:}

Horhat Răzvan Mihai

Department of Endodontics, Faculty of Dental Medicine,

"Victor Babes" University of Medicine and Pharmacy

Timisoara, Romania,

TADERP Research Center

Bv. Revolutiei din 1989, nr. 9

E-mail: razvanhorhat@yahoo.com 